\title{
TIME AND DOSE-DEPENDENT EFFECTS OF CHONDROITINASE ABC ON GROWTH OF ENGINEERED CARTILAGE
}

\author{
G.D. O’Connell'1, R.J. Nims², J. Green³, A.D. Cigan², G.A. Ateshian² and C.T. Hung ${ }^{2, *}$ \\ ${ }^{1}$ Department of Mechanical Engineering, University of California, Berkeley, CA, USA \\ ${ }^{2}$ Department of Biomedical Engineering, Columbia University, New York, NY, USA \\ ${ }^{3}$ Department of Orthopaedic Surgery, St Luke's Roosevelt Hospital Center, New York, NY, USA
}

\begin{abstract}
Tissue engineering techniques have been effective in developing cartilage-like tissues in vitro. However, many scaffold-based approaches to cultivating engineered cartilage have been limited by low collagen production, an impediment for attaining native functional load-bearing tensile mechanical properties. Enzymatic digestion of glycosaminoglycans (GAG) with chondroitinase ABC (chABC) temporarily suppresses the construct's GAG content and compressive modulus and increases collagen content. Based on the promising results of these early studies, the aim of this study was to further promote collagen deposition through more frequent chABC treatments. Weekly dosing of chABC at a concentration of $0.15 \mathrm{U} / \mathrm{mL}$ resulted in a significant cell death, which impacted the ability of the engineered cartilage to fully recover GAG and compressive mechanical properties. In light of these findings, the influence of lower chABC dosage on engineered tissue $(0.004$ and $0.015 \mathrm{U} / \mathrm{mL})$ over a longer duration (one week) was investigated. Treatment with $0.004 \mathrm{U} / \mathrm{mL}$ reduced cell death, decreased the recovery time needed to achieve native compressive mechanical properties and GAG content, and resulted in a collagen content that was $65 \%$ greater than the control. In conclusion, the results of this study demonstrate that longer chABC treatment (one week) at low concentrations can be used to improve collagen content in developing engineered cartilage more expediently than standard chABC treatments of higher chABC doses administered over brief durations.
\end{abstract}

Keywords: Cartilage tissue engineering, chondroitinase $\mathrm{ABC}$, collagen, glycosaminoglycans, articular cartilage, enzymatic digestion, dose dependence.

\footnotetext{
*Address for correspondence:

Clark T. Hung, Ph.D.

Columbia University

Biomedical Engineering Department

351 Engineering Terrace

New York

NY 10027, USA
}

Telephone Number: 212-854-6542

FAX Number: 212-854-8725

Email: cth6@columbia.edu

\section{Introduction}

Articular cartilage is the load bearing soft tissue of diarthrodial joints, and mechanical loading maintains the integrity of the tissue. The predominant extracellular matrix (ECM) constituents, proteoglycans and collagen, allow cartilage to support the high compressive and tensile loads experienced during diurnal loading. Tissue engineering techniques have been effective in developing cartilage-like tissues in vitro (Asanbaeva et al., 2008; Bian et al., 2009b; Huang et al., 2010; Johnstone et al., 2013; Lima et al., 2007; Moutos and Guilak, 2010; Nguyen et al., 2012; O'Connell et al., 2012; Sampat et al., 2011). Our prior studies have been successful in cultivating engineered cartilage constructs that have compressive mechanical properties and glycosaminoglycan (GAG) content near native values (Bian et al., 2009a; Lima et al., 2007; Sampat et al., 2011). However, these studies as well as the majority of those in the literature which feature cells embedded in a scaffold have been limited by low collagen production, an impediment for attaining native functional load-bearing tensile mechanical properties (O'Connell et al., 2012; Riesle et al., 1998). Interestingly, the collagen content within scaffoldless culture systems tends to exceed that of systems with a scaffold, while the GAG content of such systems tends to be lower than native cartilage (Huey et al., 2012).

The remarkable degree of resilience and deformability in native cartilage is due to the high density of both collagen (10-20\%/ww) and proteoglycans (4-6\%/ww) (Klein et al., 2007). Under physiological loading, cartilage experiences both high compressive strains (10-15\%) and tensile strains $(\sim 6 \%)$ in the superficial layer, which results as the two articulating surfaces contact one another (Canal et al., 2008; Chahine et al., 2004; Guterl et al., 2009; Miller and Morgan, 2010; Zhang et al., 2005). In native cartilage, these large tensile strains are supported predominantly through the tissue's dense fibrillar collagen network. Presently, engineered cartilage, both scaffold and scaffoldless systems, lack the tensile robustness of native tissue (approximately $20 \mathrm{MPa}$ ) (Park and Ateshian, 2006). Increasing the collagen content of engineered cartilage has been correlated with improvements in tensile mechanical properties (Kelly et al., 2013; Natoli et al., $2009 \mathrm{~b}$ ). Therefore, it is likely that ensuring engineered tissues can withstand the physiologic loading conditions will require recapitulating the native tissue's collagen architecture and composition (Adeeb et al., 2004; Canal et al., 2008; Chahine et al., 2004; Guterl et al., 2009).

Previously we, and others, have demonstrated that enzymatic digestion of mature engineered cartilage 
constructs can be used to improve relative collagen production (Bian et al., 2009b; Natoli et al., 2009a; Natoli et al., 2009b; Ng et al., 2009; Responte et al., 2012). Chondroitinase $\mathrm{ABC}$ (chABC) is more typically used to promote cartilage graft-host tissue integration (e.g., Quinn and Hunziker, 2002), through enhancing cell attachment to extracellular matrix. It should be noted that recruitment of synovium-derived stem cells to a chondral defect site is improved by treating the joint with a high dose of chABC $(0.1 \mathrm{U} / \mathrm{mL})$ in situ (Lee et al., 2000). chABC cleaves chondroitin sulphate, dermatan sulphate, and hyaluronic acid, affecting proteoglycans such as aggrecan and decorin. As the cleaved proteoglycans diffuse from engineered cartilage constructs and are lost into the media, there is a temporary suppression of the construct's GAG content and compressive modulus as well as a concomitant increase in collagen content (Bian et al., 2009b; Natoli et al., 2009a; Natoli et al., 2009b; Ng et al., 2009; Responte et al., 2012). Asanbaeva et al. (2008) demonstrated a threefold increase in tensile mechanical properties of cartilage constructs following chABC treatment. Furthermore, collagen fibres are thicker following enzymatic digestion, which may suggest that the deposited collagen after digestion undergoes maturation and fibril growth with less hindrance than the collagen in undigested constructs (Natoli et al., 2009a).

Although most uses of chABC treatment on engineered cartilage have been limited to single dose treatments, previous work has suggested that two treatments can be used to further increase collagen content and tensile properties (Bian et al., 2009b; Natoli et al., 2009a). However, these studies applied a second treatment after a two- to four-week recovery period following the first chABC treatment, significantly extending the culture time needed to develop mature engineered cartilage. Full recovery of GAG and compressive mechanics can require up to an additional six weeks in culture (Bian et al., 2009b). Based on the promising results of these early studies with chABC, this study aims to promote further extracellular matrix and collagen deposition through more frequent chABC treatments.

Our prior studies utilised a cell-based strategy to engineer articular cartilage by encapsulating cells in a clinically-relevant agarose hydrogel (Selmi et al., 2008). An ideal biological replacement strategy would use a biodegradable scaffold; however, agarose is a nonbiodegradable material. Degradation of agarose hydrogel with agarase has demonstrated temporary suppression of compressive mechanical properties and GAG content with an associated increase in collagen content, similar to the effects observed with chABC treatment (Bian et al., 2009b). However, for this study, the aim was to better understand dosage- and time-dependent effects of chABC on engineered cartilage development, as this may provide a more generally applicable strategy for increasing collagen content in both scaffold and scaffold-free culture systems.

The optimal dose and duration of chABC treatment for improving the biochemical and mechanical properties of engineered cartilage are not well understood. Our previous work used a chABC concentration of $0.15 \mathrm{U} / \mathrm{mL}$ applied for $48 \mathrm{~h}$, digesting approximately $85 \%$ of the deposited
GAG in engineered constructs (Bian et al., 2009b). The objective of this study was twofold: first, to evaluate the effects of one or four weekly 2-day doses of $0.15 \mathrm{U} /$ $\mathrm{mL}$ chABC on engineered cartilage; second, to evaluate the effect of very low doses of chABC supplementation during a single longer culture period (7-day dose at 0.004 and $0.015 \mathrm{U} / \mathrm{mL}$ ). The first hypothesis was that multiple 2-day doses would produce more collagen deposition than a single 2-day dose. The second hypothesis was that a 7-day dose at lower concentration would be less harmful to cells than a single 2-day dose at higher concentration, thereby producing improved matrix deposition and mechanical properties.

\section{Materials and Methods}

\section{Cell harvest and construct culture}

Chondrocytes were isolated from articular cartilage of bovine calf (3-6 week old) joints (carpometacarpal in study 1 and knee in study 2) digested with type IV collagenase in high glucose Dulbecco's Modified Eagle's Medium (hgDMEM) (Invitrogen Co., Carlsbad, CA, USA) supplemented with $5 \%$ foetal bovine serum (Invitrogen), amino acids, buffering agents, and $1 \%$ antibiotic-antimycotic (Invitrogen) at $37{ }^{\circ} \mathrm{C}$ on an orbital shaker. Primary (study 1) or passaged (study 2, defined below) cells were encapsulated in $2 \%$ agarose (type VII-A; Sigma-Aldrich, St. Louis, MO, USA) at a density of $30 \times 10^{6}$ cells $/ \mathrm{mL}$ and constructs were cored from the cellagarose mixture (Ø4 $\mathrm{mm} \times 2.3 \mathrm{~mm}$ thick) and cultured in chemically-defined, chondrogenic media (CM: hgDMEM with $0.1 \mu \mathrm{M}$ dexamethasone, $50 \mu \mathrm{g} / \mathrm{mL}$ L-proline, $50 \mu \mathrm{g} / \mathrm{mL}$ ascorbate 2-phosphate, $100 \mu \mathrm{g} / \mathrm{mL}$ sodium pyruvate (SigmaAldrich), $1 \times$ ITS + premix (Becton Dickinson, Sparks, MD, USA), $100 \mathrm{U} / \mathrm{mL}$ penicillin, and $100 \mathrm{mg} / \mathrm{mL}$ streptomycin and amphotericin $\mathrm{B}$ (Invitrogen) ) at $37^{\circ} \mathrm{C}$ under $5 \% \mathrm{CO}_{2}$. Media were supplemented with $10 \mathrm{ng} / \mathrm{mL}$ transforming growth factor beta-3 (TGF- $\beta 3$; R\&D Systems, Minneapolis, MN, USA) for the first $14 \mathrm{~d}$ of culture (Lima et al., 2007). Based on our prior work which suggested that chABC treatment can be deleterious for constructs when administered before day 14, chABC treatment was initiated on day 14, concurrently with TGF- $\beta 3$ withdrawal (Bian et al., 2009b).

\section{Study 1 - Weekly supplementation of chABC}

Study 1 examined the influence of a single or multiple weekly 2-day chABC treatments on the biochemical composition and mechanical properties of engineered cartilage. Starting on day 14, constructs were treated with $0.15 \mathrm{U} / \mathrm{mL}$ chABC (Sigma-Aldrich) for two consecutive days per week. Constructs ( $n=4$ per group) received chABC supplementation in the culture media either once (group $\mathrm{CABC} 1$; days 14-16) or four times (group CABC2; days 14-16, 21-23, 28-30 and 35-37; Fig. 1A). A corresponding control received no chABC treatment. Media were changed three times per week and constructs were cultured for $77 \mathrm{~d}$.

\section{Study 2 - Low-dose supplementation of $\operatorname{ch} A B C$}

To assess the transient influence of sustained chABC exposure to engineered cartilage, a preliminary study was 
performed in which mature chondrocyte-seeded constructs (compressive Young's modulus $\mathrm{E}_{\mathrm{Y}}=185.9 \pm 51.8 \mathrm{kPa}$, $n=3$; day 42 from casting) were cultured for $11 \mathrm{~d}$ without refreshing the media. Constructs were cultured with media supplemented with $0.15 \mathrm{U} / \mathrm{mL}$ of chABC on day 0 . Control constructs were not supplemented with chABC. On days 1 , 4, 8 and 11 samples were removed to measure GAG content using the 1,9-dimethylmethylene blue (DMMB) assay ( $n=5$ per group per time point) (Farndale et al., 1986).

Study 2 examined the effects of very low doses of chABC delivered over a single 7-day culture period. Chondrocytes were harvested from juvenile bovine knee joints and passaged (P2) in hgDMEM supplemented with $10 \%$ serum, $1 \% 100 \mathrm{U} / \mathrm{mL}$ penicillin, $100 \mathrm{mg} /$ $\mathrm{mL}$ streptomycin and amphotericin B, $1 \mathrm{ng} / \mathrm{mL}$ TGF $\beta 1$, $10 \mathrm{ng} / \mathrm{mL}$ platelet-derived growth factor (PDGF- $\beta \beta$, from Invitrogen), and $5 \mathrm{ng} / \mathrm{mL}$ basic fibroblast growth factor (bFGF2) (Sampat et al., 2011). Constructs were then cast using passaged chondrocytes. Study 2 was also repeated with primary chondrocytes and similar observations were observed; therefore, only the results from the passaged constructs are reported here.

At day 14, constructs were divided into four groups, with three groups receiving chABC. In one group, the culture medium was supplemented at the same dosage as in Study 1, $0.15 \mathrm{U} / \mathrm{mL}$ of chABC for two days (high-dose group) (Bian et al., 2009b). Two other groups received a lower dose of chABC, which represented $2.5 \%$ (0.004 U/ $\mathrm{mL})$ and $10 \%(0.015 \mathrm{U} / \mathrm{mL})$ of the high-dose group over a week-long supplementation period (Fig. 1B). Fresh chABC of these two groups was supplied during each media change between days 14 and 21 (3 media changes per week). Mechanical and biochemical properties were assessed at days 14 and 56 ( $n=5-7$ per group).

\section{Mechanical and biochemical characterisation}

$\mathrm{E}_{\mathrm{Y}}$ was determined using unconfined compression stressrelaxation at $10 \%$ strain. The dynamic modulus was measured from the response to a superposed sinusoidal input of $\pm 1 \%$ strain at $0.5 \mathrm{~Hz}$. Prior to biochemical analysis, construct wet weight was measured. Swelling ratio (SR) was computed by normalising the current day's wet weight by the average wet weight on day 0 for each study. Samples were digested with proteinase K (MP Biomedical, Santa Ana, CA, USA) as previously described (Hollander et al., 1994). DNA content was determined using the PicoGreen Kit (Invitrogen). GAG and collagen contents were determined using the DMMB and hydroxyproline assays, respectively (Farndale et al., 1986; Hollander et al., 1994). ANOVA were performed (Study 1: factors were day and treatment; Study 2: factor was treatment) on mechanical and biochemical properties. When significance was observed, a Bonferroni-corrected post-hoc test of the means was performed. Significance was set at $p \leq 0.05$. Data are presented as mean \pm standard deviation.

\section{Results}

\section{Study 1}

Undigested control constructs developed $\mathrm{E}_{\mathrm{Y}}$, GAG concentration, and collagen concentration similar to

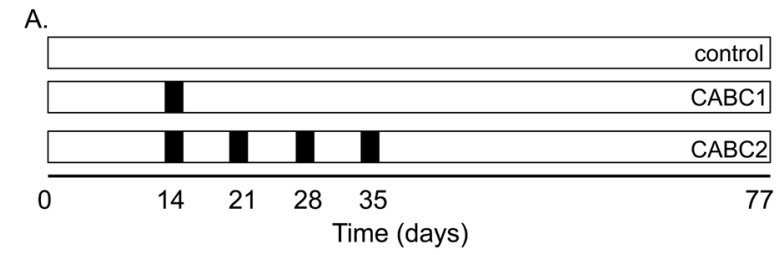

B.

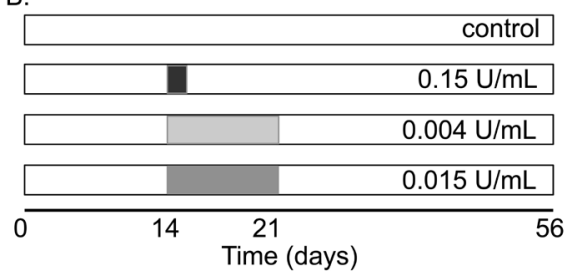

Fig. 1. Schematic of study design for (A) Study 1 and (B) Study 2. In Study 1, groups were differentiated by the number of weeks during which samples received chABC supplementation. In Study 2, groups were differentiated by the dosage of chABC, which was administered over a single week. Shaded regions represent the duration (time) and intensity (dosage) of chABC administration.

previous studies (Fig. 2A,C,D) (Byers et al., 2008; Lima et al., 2007). Both $\mathrm{E}_{\mathrm{Y}}$ and dynamic modulus were diminished to near day 0 levels following chABC treatments on day 14 ( $p<0.05$; Fig. 2A,B, respectively); from these near zero levels, $\mathrm{E}_{\mathrm{Y}}$ and dynamic modulus in the $\mathrm{CABC} 1$ group recovered to control levels by day 77 (Fig. 2A,B; $p=0.98)$. In contrast, $\mathrm{E}_{\mathrm{Y}}$ and dynamic modulus in the $\mathrm{CABC} 2$ group failed to recover to control levels over the following 6 weeks. Similarly, GAG concentration (normalised to current day wet weight) was similar to control values in the $\mathrm{CABC} 1$ group (Fig. $2 \mathrm{C} ; p=0.98$ ), but was different between the control and $\mathrm{CABC} 2$ group on day $77(p<0.05)$. Collagen levels (normalised to current day wet weight) were similar across all three groups for all time points (Fig. 2D; $p=0.98$ ). After $77 \mathrm{~d}$ of culture, the cell content per construct was similar between the control and $\mathrm{CABC} 1$ group (Fig. 2E; $p=0.52$ ), but the cell count per construct of the $\mathrm{CABC} 2$ group was significantly lower than the control $(p<0.05)$.

There was an observable and significant difference in the expansion and physical growth between the treatment groups of this study. Construct wet weight on day 0 was $24.4 \pm 0.3 \mathrm{mg}$. By day 77, tissue swelling in the control group was significantly higher than both chABC-treated groups (Fig. 3A; SR $\sim 3 ; p<0.001$ ). Furthermore, the $\mathrm{SR}$ of CABC1 (nearly twofold increase in wet weight) was significantly higher than that of $\mathrm{CABC} 2(\mathrm{SR} \sim 1$; $p<0.001)$. Due to these significant differences in construct wet weight, GAG and collagen contents were also analysed when normalised by the initial (day 0 ) construct wet weight (Fig. 3B,C). This normalisation provided a more accurate measure of the total ECM synthesis per construct. When the GAG content is normalised to the reference (day 0) configuration, one chABC treatment significantly decreased GAG content from the untreated 

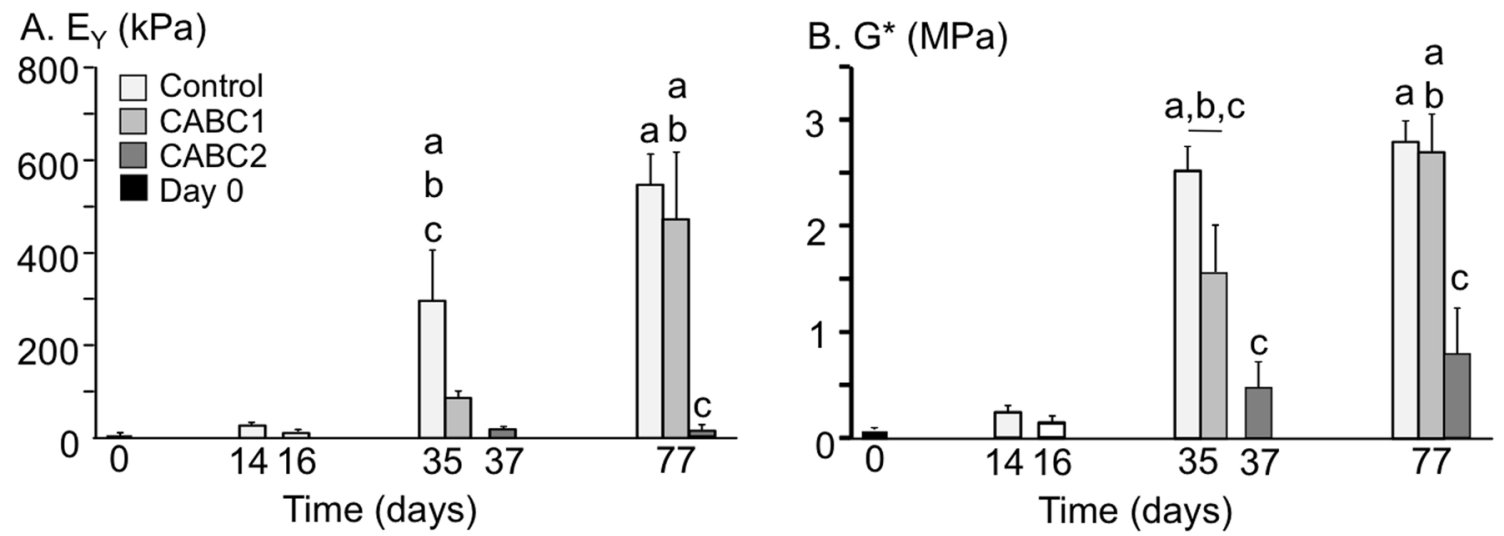

C. GAG / WW (\%)

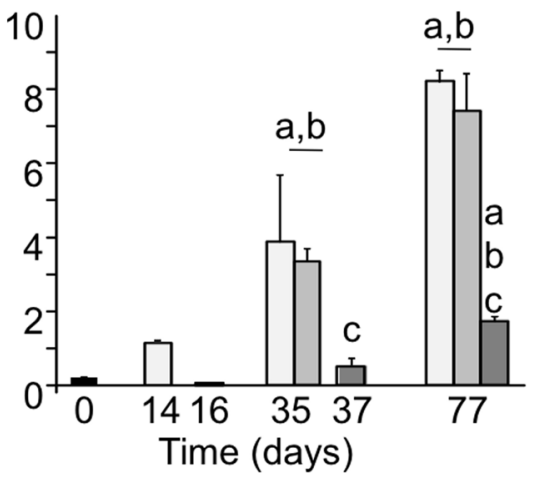

D. Collagen / WW (\%)

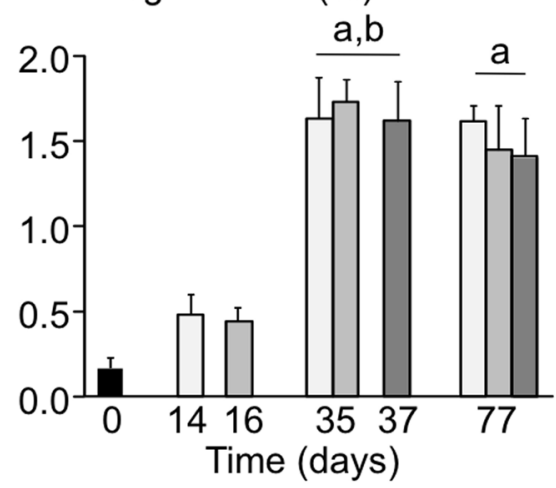

E. Cell Count

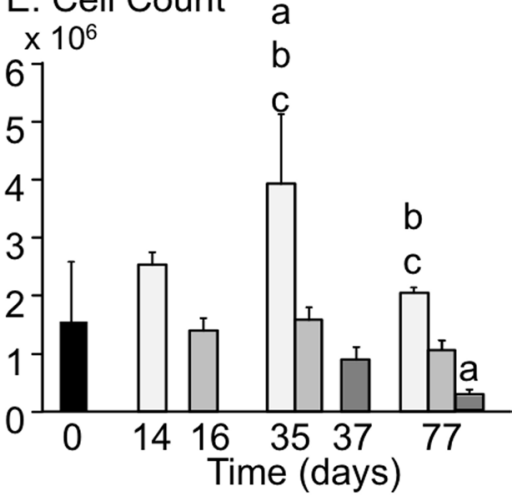

Fig. 2. Mechanical and biochemical properties of constructs from Study 1: (A) $E_{\gamma},\left(\right.$ B) $G^{*}$, (C) GAG concentration relative to current day wet weight, (D) collagen concentration relative to current day wet weight, and (E) total number of cells within the construct. ' $\mathrm{a}$ ' denotes difference from day $0(p<0.05)$; ' $\mathrm{b}$ ' denotes difference from previous time point $(p<0.05)$; 'c' denotes difference from other groups at time point $(p<0.05)$.
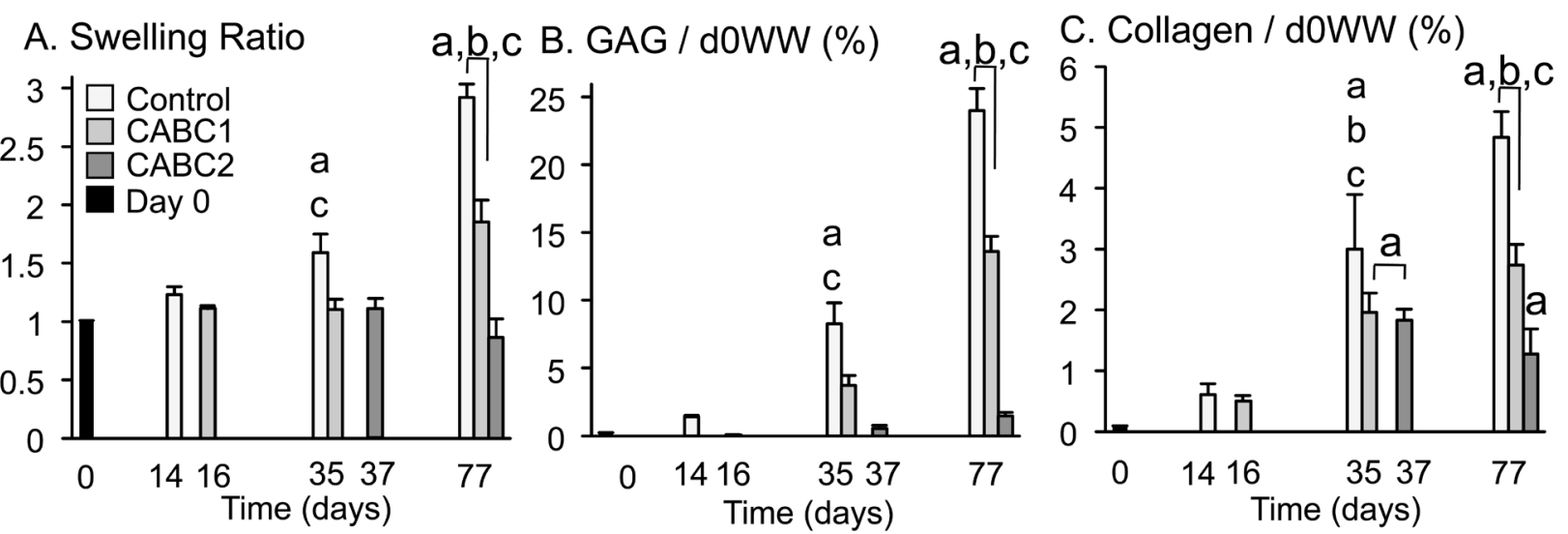

Fig. 3. The (A) swelling ratio, calculated as current wet-weight divided by day 0 wet weight, and biochemical properties (B) GAG and (C) collagen normalised to the construct wet weight at day 0 . The day 0 wet weight for study 1 was $24.4 \pm 0.3 \mathrm{mg}$. 'a' denotes difference from day $0(p<0.05)$; ' $\mathrm{b}$ ' denotes difference from previous time point $(p<0.05)$; 'c' denotes difference from other groups at time point $(p<0.05)$.

control (Fig. 3B; $p<0.001$ ); there was a further decline in total construct $\mathrm{GAG}$ with increasing the number of chABC treatments (Fig. 3B; $p<0.001$ ). Similarly, total collagen content decreased with multiple chABC treatments (Fig. 3C; $p<0.001)$.

\section{Study 2}

Preliminary work for Study 2 investigated the effect of sustained chABC activity following a single treatment application $(0.15 \mathrm{U} / \mathrm{mL})$. The GAG content of chABCdigested constructs continued to decrease through day 8 (Fig. 4), at which point, chABC activity decreased, which 
is comparable to previously reported chABC activity (Lee et al., 2010). The GAG content for the control constructs did not change over the 11-day culture period, which is consistent with GAG data previously reported for mature engineered cartilage constructs (Bian et al., 2009b). For Study 2, low-dose chABC treatments were applied fresh during each media change between days 14 and 21 . The results from the preliminary study confirm chABC enzyme activity between media changes.

At day 56, gross images of the engineered cartilage demonstrate differences in final dimensions (Fig. 5A). The diameter and thickness of control constructs were greater than the dimensions of chABC-treated constructs $(p<0.01)$. The final thickness of the $0.004 \mathrm{U} / \mathrm{mL}$ group was $2.72 \pm 0.10 \mathrm{~mm}$, significantly lower than the control group $(p<0.01)$. Similar to the results in Study 1, the swelling ratio of chABC-treated constructs was significantly lower than the control ( $p<0.001 ;$ Fig. 5B). Construct wet weight of undigested control constructs on day 56 was 52.02 $\pm 1.46 \mathrm{mg}$ and was approximately twofold higher than the wet weight on day 0 of $26.6 \pm 0.6 \mathrm{mg}$.

$\mathrm{E}_{\mathrm{Y}}$ of the control group on day 56 was $603 \pm 62 \mathrm{kPa}$ (Fig. 6A). By the completion of the recovery period on day 56 , only the $0.004 \mathrm{U} / \mathrm{mL}$ group had an $\mathrm{E}_{\mathrm{Y}}$ comparable to the undigested control, while $\mathrm{E}_{\mathrm{Y}}$ of the $0.015 \mathrm{U} / \mathrm{mL}$ group was approximately $50 \%$ of the day 14 values (Fig. 6A). The dynamic moduli of the $0.15 \mathrm{U} / \mathrm{mL}$ and $0.004 \mathrm{U} / \mathrm{mL}$ groups were $25-30 \%$ greater than the undigested control while the modulus for the $0.015 \mathrm{U} / \mathrm{mL}$ treatment was significantly lower than the undigested control ( $p<0.02$; Fig. $6 \mathrm{~B})$.

The DNA content for chABC-digested constructs was lower at day 56 than the control group at day 14, with the $0.15 \mathrm{U} / \mathrm{mL}$ group having the largest decrease in DNA content (Fig. 7A). The DNA content of all three chABC groups stayed above the initial day 0 level, which was not observed with multiple high-dose treatments in Study 1 (Fig. 2E - CABC2). The GAG content normalised by DNA for the $0.004 \mathrm{U} / \mathrm{mL}$ and the $0.15 \mathrm{U} / \mathrm{mL}$ group was

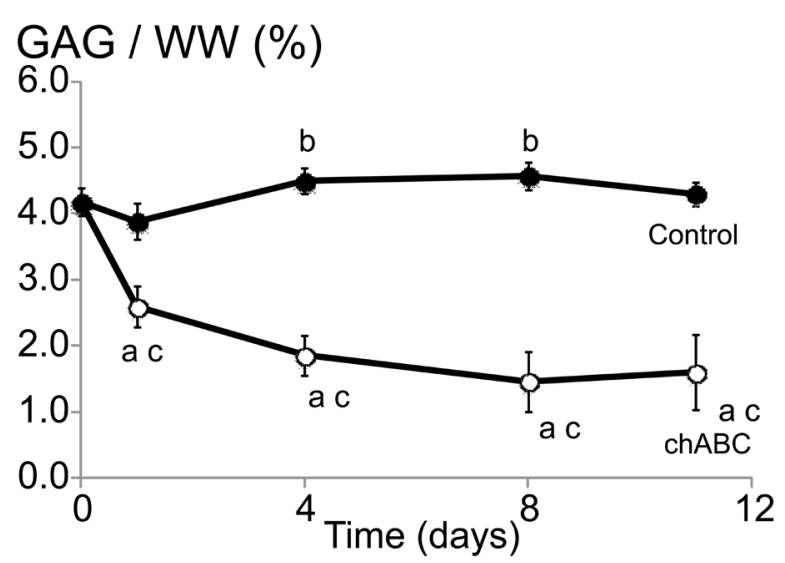

Fig. 4. GAG content normalised by wet weight of mature chondrocyte-seeded constructs (cultured for 42 days) cultured for an additional 11 days in chemicallydefined media (control) or media supplemented with $0.15 \mathrm{U} / \mathrm{mL}$ chABC on day 0 . 'a' denotes $p<0.05$ between control and chABC group at each time point; ' $\mathrm{b}$ ' denotes $p<0.05$ for the control group versus day 0 values; 'c' denotes $p<0.05$ for the chABC group versus day 0 values.

more than $2 \times$ greater than the control. The GAG content normalised by current day wet weight was $9.5 \pm 0.7 \%$ / ww for the $0.004 \mathrm{U} / \mathrm{mL}$ group and was $20 \%$ higher than the control $(7.8 \pm 1.5 \% / \mathrm{ww} ; p=0.04$; Fig. $7 \mathrm{~B})$. The GAG content normalised by wet weight for the $0.15 \mathrm{U} / \mathrm{mL}$ group was not significantly different from the control group. To account for the differences in swelling ratio with chABC treatment (Fig. 5B), the GAG content was also normalised by the day 0 wet weight. Only the $0.015 \mathrm{U} / \mathrm{mL}$ group had a GAG content normalised by day 0 wet weight that was significantly lower than the control ( $p<0.0001$; Fig. 8A).

By day 56, the $0.004 \mathrm{U} / \mathrm{mL}$ group had the highest collagen content $(3.25 \pm 0.37 \% / \mathrm{ww}$ and $21.0 \pm 2.3 \%$ /dry
Fig. 5. (A) Images of representative samples from day 56 for untreated and chABC-treated constructs. Bar $=1 \mathrm{~mm}$. For Study 2, on day 0 , the construct diameter was 4.00 $\pm 0.09 \mathrm{~mm}$ and the wet weight was $26.6 \pm 0.6 \mathrm{mg}$. (B) Swelling ratio of constructs at day 56. The initial wet weight of constructs was $26.6 \pm 0.6$ $\mathrm{mg}$ at day 0 . 'a' denotes $p<0.05$ versus control.
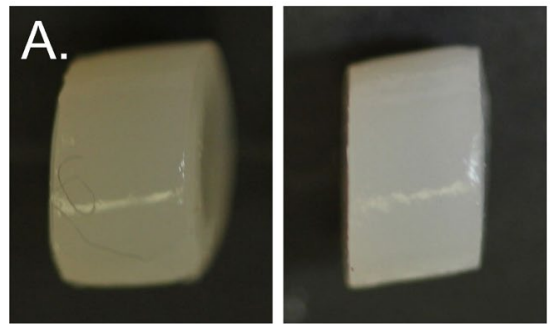

Control

$0.15 \mathrm{U} / \mathrm{mL}$

B. Swelling Ratio

2.0

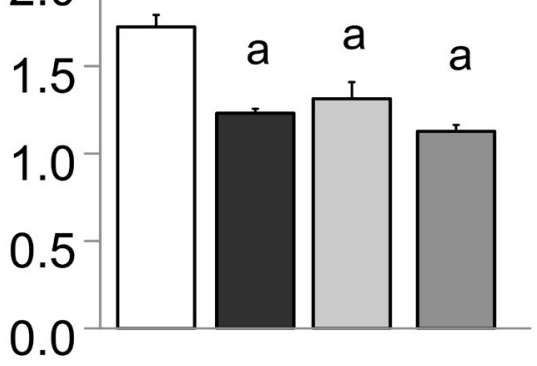

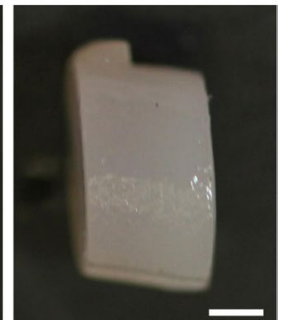

$0.015 \mathrm{U} / \mathrm{mL}$

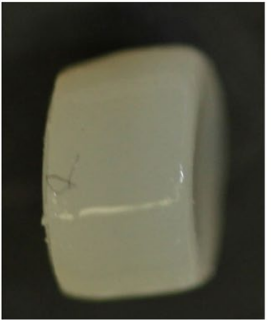

$0.004 \mathrm{U} / \mathrm{mL}$ 


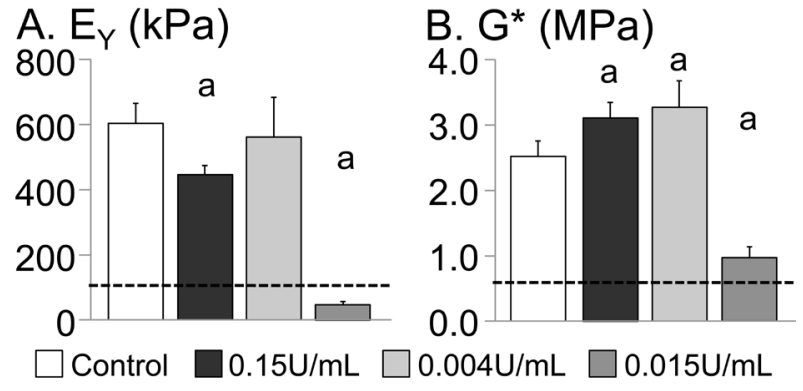

Fig. 6. Compressive mechanical properties of constructs from Study 2 on day 56. (A) Equilibrium Young's modulus and (B) dynamic modulus measured at $0.5 \mathrm{~Hz}$. Mechanical properties of the very low chABC group $(0.004 \mathrm{U} / \mathrm{mL}$ for one week) recovered faster than digesting constructs with a higher single dose of chABC $(0.15 \mathrm{U} / \mathrm{mL}$ applied on day 14). 'a' denotes significant differences from control. The dotted line represents day 14 values (i.e. pre-digestion values).

weight), $65 \%$ higher than the control ( $p<0.001$; Fig. $7 \mathrm{C}$ ). The collagen content of the $0.004 \mathrm{U} / \mathrm{mL}$ and the $0.15 \mathrm{U} /$ $\mathrm{mL}$ groups was significantly greater than the control for all normalisation methods (i.e. normalisation by DNA, wet weight or dry weight; $p<0.02$; Figs. $7 \mathrm{C}$,D and $8 \mathrm{~B}$ ). The total construct collagen content of the $0.004 \mathrm{U} / \mathrm{mL}$ group was $40 \%$ greater than the control $(1.43 \pm 0.16 \mathrm{mg}$ for the $0.004 \mathrm{U} / \mathrm{mL}$ group and $1.01 \pm 0.23 \mathrm{mg}$ for the control; $p<0.01)$.

\section{Discussion}

Recapitulating the biochemical composition and structure of native cartilage remains a significant challenge for the field of tissue engineering. Chondroitinase ABC (chABC) has been used successfully to improve collagen fibre structure and composition in engineered cartilage (MacBarb et al., 2013; Natoli et al., 2009b; Responte et al., 2012). Accumulation of GAG occurs much faster than collagen in our engineered constructs, such that the GAG content at day 56 is approximately $3 x$ greater than the collagen content whereas in native tissue there is $2 x$ more collagen than GAG. Previous studies have suggested that excessive GAG deposition early in the culture of engineered cartilage may inhibit collagen deposition due to overcrowding (Bian et al., 2009b). Enhancing the collagen content of replacement tissues is believed to be a necessary condition for developing the high tensile mechanical properties present in native cartilage. Such tensile robustness will be crucial for replacement tissues to withstand the deformation from the high compressive loads within the normal loading of the articular cartilage (Treppo et al., 2000). As the fibrillar collagen network provides nearly all of the tensile support in native cartilage, the aim of this study was to test the hypothesis that more frequent chABC supplementation can improve collagen deposition.
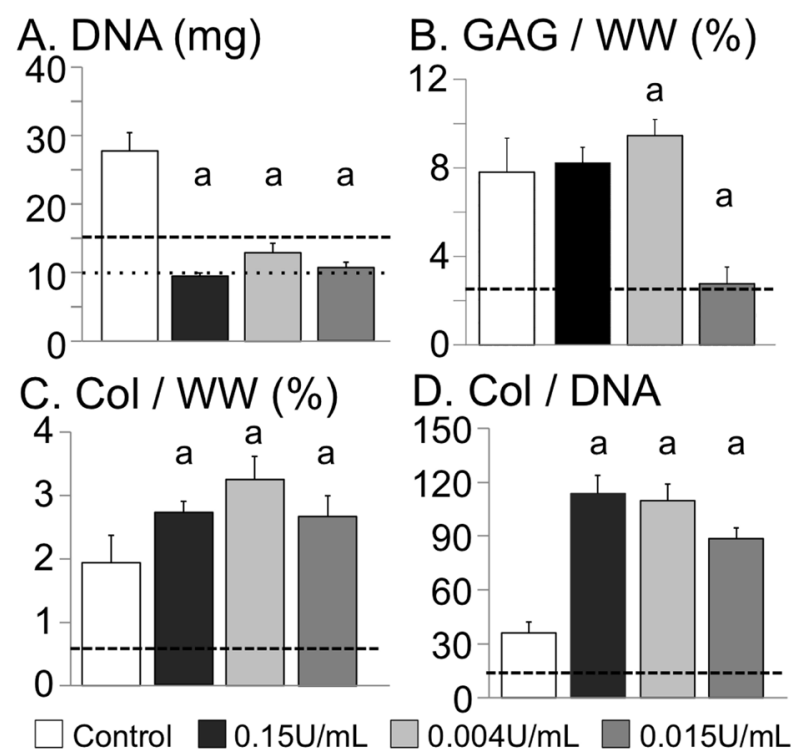

Control

$0.15 \mathrm{U} / \mathrm{mL}$

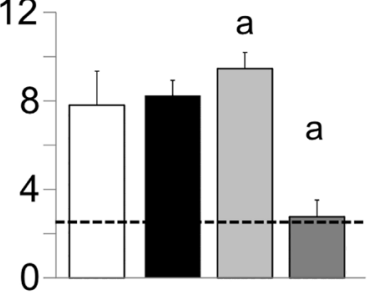

Fig. 7. Day 56 biochemical properties of engineered cartilage from Study 2. (A) DNA content, (B) GAG content normalised by current day wet weight (i.e. day 56), (C) collagen normalised by current day wet weight, and (D) collagen content normalised by DNA content. 'a' denotes significant differences from control. The dotted line in (A) represents the day 0 DNA level and the dashed line in (A), (B), (C) and (D) represents day 14 values (i.e. predigestion values).

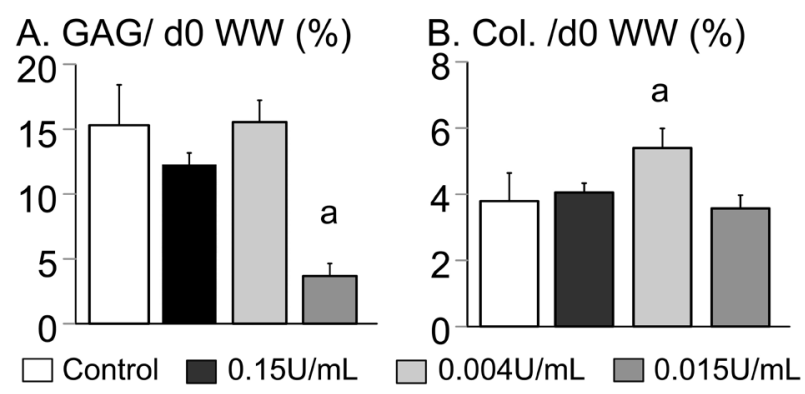

Fig. 8. Biochemical properties of engineered cartilage from Study 2. (A) GAG content and (B) collagen content normalised by day 0 wet weight (WW) at day 56. ' $a$ ' denotes significant differences from control.

Although prior investigations of a single treatment of chABC caused some cell death, this effect was not significant enough to prevent ECM deposition, allowing for full recovery of mechanical properties and GAG when normalised to the tissue wet weight at the end of the study (Bian et al., 2009b; Natoli et al., 2009a; O'Connell et al., 2012; Responte et al., 2012; Treppo et al., 2000). Based on this prior work, it was hypothesised that by increasing the duration of weekly chABC administrations, collagen deposition would be further enhanced. However, the findings of this study demonstrated detrimental effects of multiple chABC treatments on long-term cell proliferation. Among the various protocols tested in Study 1 and Study 2 , only the low dose of chABC supplementation of Study 2 was effective in increasing the collagen content, producing 
the least cell death following treatment, and decreasing the culture time needed to recover mechanical properties and GAG content.

The results of Study 1 indicated that multiple high-dose chABC treatments $(0.15 \mathrm{U} / \mathrm{mL})$ had an additive detrimental effect on cell viability, causing unrecoverable degradation of the construct ECM, mechanical properties and swelling ratio (Figs. 2 and 3). Interestingly, treatment groups with similar GAG levels (as normalised by current day wet weight) also had similar compressive mechanical properties (notably day 77 of the control and CABC1 groups in Fig. 2). This similarity is in line with previous work indicating that the fixed-charge density of cartilage (due to the high GAG content) contributes strongly to the compressive mechanical properties (Eisenberg and Grodzinsky, 1985; Lai et al., 1991). As a better representation of the loss of ECM synthesis, biochemical composition data were normalised to the initial construct wet weight (i.e. day 0 values; Fig. 3). These results illustrated the severe reduction of both collagen and GAG elaboration when constructs were treated with a single high-dose of chABC. Such results are in line with our earlier work, which demonstrated that constructs supplemented with chABC during the first four weeks of culture had a significantly reduced ECM synthesis capacity (Bian et al., 2009b). This earlier work also observed a reduction in cell viability in groups treated for prolonged periods with high-dose treatments of chABC, potentially contributing to the drastically reduced ECM levels. Taken together, both this earlier work and Study 1 suggest that increasing chABC dosage and frequency can be deleterious for engineered construct growth.

In light of these findings, in Study 2 we attempted to limit the decrease in DNA content following multiple chABC applications by utilising very low doses of chABC. Applying a very low concentration of chABC (i.e. less than $3 \%$ of the high-dose group; Study $2-0.004 \mathrm{U} / \mathrm{mL}$ group) over a one-week culture period was successful in increasing collagen production without causing a detrimental amount of cell death. At day 56, the DNA content of the very low dose group was greater than the single two-day high dose treatment group $(0.15 \mathrm{U} / \mathrm{mL}$ group $)$, which suggests that there is a strong correlation between chABC concentration and cell death. Together with Study 1, these results indicate that chABC is not harmless to chondrocytes, and that the concentration, duration and frequency must be balanced to improve collagen deposition, while maintaining cell proliferation, GAG production, and recovery.

The very low chABC treatment group $(0.004 \mathrm{U} / \mathrm{mL})$ demonstrated an ability to fully recover compressive mechanical properties, while improving the overall biochemical composition (Figs. 6 and 7). Although not investigated here, previous studies with engineered fibrocartilage tissues have demonstrated an increase in fibre diameter and collagen density following chABC treatment (MacBarb et al., 2013; Responte et al., 2012). Importantly, the collagen content (normalised to day 56 wet weight, DNA, and total mass within the construct) of the very low dose chABC group was $65 \%$ greater than the control group, compared to a $40 \%$ increase in collagen content with the single high-dose chABC treatment (Figs. 7C, D).
These findings suggest that a very low dose of chABC may be more beneficial than a single high-dose treatment for decreasing the total culture time, as well as increasing the overall biochemical composition and tensile mechanical properties of engineered cartilage (Kelly et al., 2013).

Previous studies have used multiple high-dose chABC treatments on engineered cartilage to improve collagen production without fully compromising the mechanical integrity of the tissue (Bian et al., 2009b; Natoli et al., 2009a). In those studies, the second chABC treatment occurred two to four weeks after the first treatment. It is likely that allowing for sufficient extracellular matrix deposition following the first treatment helps to protect the cells during the second enzymatic treatment. The results of Study 2 show that a low-dose protocol eliminates the need for a long recovery time between treatments, which is ideal for developing engineered cartilage as a clinical repair strategy.

Although the collagen content of the very low dose group was $65 \%$ higher than undigested control $(3.3 \%$ / Ww at day 56), it should be noted that the collagen content of native cartilage is $10-15 \% / \mathrm{ww}$ (Klein et al., 2007; O'Connell et al., 2012; Treppo et al., 2000). Therefore, we were able to cultivate engineered cartilage with collagen composition that was approximately $30 \%$ of native values, demonstrating the challenge of recapitulating collagen composition in engineered cartilage in vitro. Obtaining native collagen levels may require multiple low-dose treatments over an extended culture duration, particularly for application to engineered human tissues, where the low GAG and collagen synthesis rates typical of adult and often osteoarthritic chondrocytes will further confound obtaining native properties. Furthermore, the collagen alignment of native cartilage tissue is depth dependent, while the collagen alignment of engineered cartilage is random under free-swelling conditions (Bian et al., 2009b). Mechanical stimulation has been shown to preferentially align collagen fibres along the positive principle directions of strain (Kelly et al., 2006). It is likely the techniques used here will need to be combined with other culture techniques, such as applied physical stimuli (Kock et al., 2013; Mauck et $a l ., 2000)$, to develop engineered tissues that replicate the collagen composition and structure of native tissue, and these investigations are the topics of ongoing studies.

\section{Conclusion}

Enzymatic digestion of over-deposited GAG with chABC can be an effective method to improve collagen composition in engineered cartilage. This study demonstrated that the concentration and duration of chABC treatments can be optimised to improve collagen deposition and decrease the total culture time needed in vitro, while limiting cell death due to enzymatic treatment.

\section{Acknowledgements}

Research reported in this publication was supported by the National Institute of Arthritis and Musculoskeletal 
and Skin Diseases of the National Institutes of Health under Award Numbers R01AR060361, R01AR046568, and T32AR059038 (AD Cigan). The content is solely the responsibility of the authors and does not necessarily represent the official views of the National Institutes of Health. The authors would like to thank Michael B. Albro, Victoria Cui, and Daniel B. Park for their help with data collection. We wish to confirm that there are no known conflicts of interest associated with this publication and there has been no significant financial support for this work that could have influenced its outcome.

\section{References}

Adeeb SM, Sayed Ahmed EY, Matyas J, Hart DA, Frank CB, Shrive NG (2004) Congruency effects on load bearing in diarthrodial joints. Comput Methods Biomech Biomedical Eng 7: 147-157.

Asanbaeva A, Tam J, Schumacher BL, Klisch SM, Masuda K, Sah RL (2008) Articular cartilage tensile integrity: modulation by matrix depletion is maturationdependent. Arch Biochem Biophys 474: 175-182.

Bian L, Angione SL, Ng KW, Lima EG, Williams DY, Mao DQ, Ateshian GA, Hung CT (2009a) Influence of decreasing nutrient path length on the development of engineered cartilage. Osteoarthritis Cartilage 17: 677-685.

Bian L, Crivello KM, Ng KW, Xu D, Williams DY, Ateshian GA, Hung CT (2009b) Influence of temporary chondroitinase ABC-induced glycosaminoglycan suppression on maturation of tissue-engineered cartilage. Tissue Eng Part A 15: 2065-2072.

Byers BA, Mauck RL, Chiang IE, Tuan RS (2008) Transient exposure to transforming growth factor beta 3 under serum-free conditions enhances the biomechanical and biochemical maturation of tissue-engineered cartilage. Tissue Eng Part A 14: 1821-1834.

Canal CE, Hung CT, Ateshian GA (2008) Twodimensional strain fields on the cross-section of the bovine humeral head under contact loading. J Biomech 41: 3145 3151 .

Chahine NO, Wang CC, Hung CT, Ateshian GA (2004) Anisotropic strain-dependent material properties of bovine articular cartilage in the transitional range from tension to compression. J Biomech 37: 1251-1261.

Eisenberg SR, Grodzinsky AJ (1985) Swelling of articular cartilage and other connective tissues: electromechanochemical forces. J Orthop Res 3: 148-159.

Farndale RW, Buttle DJ, Barrett AJ (1986) Improved quantitation and discrimination of sulphated glycosaminoglycans by use of dimethylmethylene blue. Biochim Biophys Acta 883: 173-177.

Guterl CC, Gardner TR, Rajan V, Ahmad CS, Hung CT, Ateshian GA (2009) Two-dimensional strain fields on the cross-section of the human patellofemoral joint under physiological loading. J Biomech 42: 1275-1281.

Hollander AP, Heathfield TF, Webber C, Iwata Y, Bourne R, Rorabeck C, Poole AR (1994) Increased damage to type II collagen in osteoarthritic articular cartilage detected by a new immunoassay. J Clin Invest 93: 17221732.
Huang AH, Farrell MJ, Kim M, Mauck RL (2010) Long-term dynamic loading improves the mechanical properties of chondrogenic mesenchymal stem cell-laden hydrogel. Eur Cell Mater 19: 72-85.

Huey DJ, Hu JC, Athanasiou KA (2012) Unlike bone, cartilage regeneration remains elusive. Science 338: $917-$ 921.

Johnstone B, Alini M, Cucchiarini M, Dodge GR, Eglin D, Guilak F, Madry H, Mata A, Mauck RL, Semino CE, Stoddart MJ (2013) Tissue engineering for articular cartilage repair - the state of the art. Eur Cell Mater 25: 248-267.

Kelly TAN, Ng KW, Wang CCB, Ateshian GA, Hung CT (2006) Spatial and temporal development of chondrocyte-seeded agarose constructs in free-swelling and dynamically loaded cultures. J Biomech 39: 14891497.

Kelly TA, Roach BL, Weidner ZD, Mackenzie-Smith CR, O'Connell GD, Lima EG, Stoker AM, Cook JL, Ateshian GA, Hung CT (2013) Tissue-engineered articular cartilage exhibits tension-compression nonlinearity reminiscent of the native cartilage. J Biomech 46: 17841791.

Klein TJ, Chaudhry M, Bae WC, Sah RL (2007) Depthdependent biomechanical and biochemical properties of fetal, newborn, and tissue-engineered articular cartilage. J Biomech 40: 182-190.

Kock LM, Ito K, van Donkelaar CC (2013) Sliding indentation enhances collagen content and depthdependent matrix distribution in tissue-engineered cartilage constructs. Tissue Eng Part A 19: 1949-1959.

Lai WM, Hou JS, Mow VC (1991) A triphasic theory for the swelling and deformation behaviors of articularcartilage. J Biomech Eng 113: 245-258.

Lee MC, Sung KL, Kurtis MS, Akeson WH, Sah RL (2000) Adhesive force of chondrocytes to cartilage. Effects of chondroitinase ABC. Clin Orthop Relat Res 370: 286294.

Lee H, McKeon RJ, Bellamkonda RV (2010) Sustained delivery of thermostabilized chABC enhances axonal sprouting and functional recovery after spinal cord injury. Proc Natl Acad Sci USA 107: 3340-3345.

Lima EG, Bian L, Ng KW, Mauck RL, Byers BA, Tuan RS, Ateshian GA, Hung CT (2007) The beneficial effect of delayed compressive loading on tissue-engineered cartilage constructs cultured with TGF-beta3. Osteoarthritis Cartilage 15: 1025-1033.

MacBarb RF, Makris EA, Hu JC, Athanasiou KA (2013) A chondroitinase-ABC and TGF-beta1 treatment regimen for enhancing the mechanical properties of tissueengineered fibrocartilage. Acta Biomater 9: 4626-4634.

Mauck RL, Soltz MA, Wang CC, Wong DD, Chao PH, Valhmu WB, Hung CT, Ateshian GA (2000) Functional tissue engineering of articular cartilage through dynamic loading of chondrocyte-seeded agarose gels. J Biomech Eng 122: 252-260.

Miller GJ, Morgan EF (2010) Use of microindentation to characterize the mechanical properties of articular cartilage: comparison of biphasic material properties across length scales. Osteoarthritis Cartilage 18: 1051-1057. 
Moutos FT, Guilak F (2010) Functional properties of cell-seeded three-dimensionally woven poly(epsiloncaprolactone) scaffolds for cartilage tissue engineering. Tissue Eng Part A 16: 1291-1301.

Natoli RM, Responte DJ, Lu BY, Athanasiou KA (2009a) Effects of multiple chondroitinase ABC applications on tissue engineered articular cartilage. J Orthop Res 27: 949-956.

Natoli RM, Revell CM, Athanasiou KA (2009b) Chondroitinase $\mathrm{ABC}$ treatment results in greater tensile properties of self-assembled tissue-engineered articular cartilage. Tissue Eng Part A 15: 3119-3128.

Ng KW, Kugler LE, Doty SB, Ateshian GA, Hung CT (2009) Scaffold degradation elevates the collagen content and dynamic compressive modulus in engineered articular cartilage. Osteoarthritis Cartilage 17: 220-227.

Nguyen QT, Hwang Y, Chen AC, Varghese S, Sah RL (2012) Cartilage-like mechanical properties of poly (ethylene glycol)-diacrylate hydrogels. Biomaterials 33: 6682-6690.

O’Connell GD, Lima EG, Bian L, Chahine NO, Albro MB, Cook JL, Ateshian GA, Hung CT (2012) Toward engineering a biological joint replacement. J Knee Surg 25: 187-196.

Park S, Ateshian GA (2006) Dynamic response of immature bovine articular cartilage in tension and compression, and nonlinear viscoelastic modeling of the tensile response. J Biomech Eng 128: 623-630.

Quinn TM, Hunziker EB (2002) Controlled enzymatic matrix degradation for integrative cartilage repair: effects on viable cell density and proteoglycan deposition. Tissue Eng 8: 799-806.

Responte DJ, Arzi B, Natoli RM, Hu JC, Athanasiou KA (2012) Mechanisms underlying the synergistic enhancement of self-assembled neocartilage treated with chondroitinase-ABC and TGF-beta1. Biomater 33: 3187 3194.

Riesle J, Hollander AP, Langer R, Freed LE, VunjakNovakovic G (1998) Collagen in tissue-engineered cartilage: Types, structure, and crosslinks. J Cell Biochem 71: 313-327.

Sampat SR, O'Connell GD, Fong JV, Alegre-Aguaron E, Ateshian GA, Hung CT (2011) Growth factor priming of synovium-derived stem cells for cartilage tissue engineering. Tissue Eng Part A 17: 2259-2265.

Selmi TA, Verdonk P, Chambat P, Dubrana F, Potel JF, Barnouin L, Neyret P (2008) Autologous chondrocyte implantation in a novel alginate-agarose hydrogel: outcome at two years. J Bone Joint Surg Br 90: 597-604.

Treppo S, Koepp H, Quan EC, Cole AA, Kuettner KE, Grodzinsky AJ (2000) Comparison of biomechanical and biochemical properties of cartilage from human knee and ankle pairs. J Orthop Res 18: 739-748.

Zhang J, Jin GC, Meng LB, Jian LH, Wang AY, Lu SB (2005) Strain and mechanical behavior measurements of soft tissues with digital speckle method. J Biomed Opt 10: 034021 .

\section{Discussion with Reviewers}

Reviewer II: These findings are very intriguing for young bovine cells, which produce an abundance of proteoglycans in culture. Have the authors investigated human cells or adult animal cells to see if the same phenomena and responses are present?

Authors: Current studies are being performed on developing engineered cartilage constructs with human cells (both mesenchymal stem cells and articular chondrocytes). However, as the reviewer notes, GAG production from juvenile bovine cells is much more abundant than human cells. Therefore, the low level of the chABC shown to be beneficial in this study may have to be supplemented later in culture as GAG may not hinder collagen deposition until it is given sufficient time to develop within the constructs. 